\title{
Contrôle de procédés durant le micro-usinage de céramique par laser à excimère
}

\author{
C. Stauter, J. Fontaine et A. Biernaux* \\ Laboratoire d'Applications des Lasers, Ecole Nationale Supérieure des Arts et Métiers de \\ Strasbourg, 24 Bld de la Victoire, 67000 Strasbourg, France \\ * Société IREPA LASER, Parc d'Innovation d'lllkirch, 67400 Illkirch, France
}

\begin{abstract}
Characterization of the effects of the interaction between short laser pulses and a material has been made in the conditions of ceramic micromachining. Fast heating of the target surface results in a pressure wave that propagates inside the sample and a shock wave in the surrounding gas medium. By monitoring the energy of the shock wave using a laser beam deflection technique and a piezoelectric transducer, it is possible to relate the ablation rate to the observed signals. Experiments have been made using Q-switched $\mathrm{Nd}: Y A G$ and $\mathrm{KrF}$ lasers. At high fluences, in particular at 1,06 $\mu \mathrm{m}$, gas breakdown drastically alters the process conditions and disperses the recorded data. Nevertheless, we have determined the range of validity for monitoring of the process using simple detection devices.
\end{abstract}

\section{INTRODUCTION}

$\mathrm{L}^{\prime}$ utilisation des lasers $\mathrm{CO} 2$ et $\mathrm{Nd}$ :YAG de puissance pour le traitement et $\mathrm{I}^{\prime}$ usinage des matériaux a connu une forte croissance durant ces dernières années. Plus récemment encore, une autre classe de lasers, ceux pouvant délivrer des impulsions courtes, tels que les lasers à solide déclenchés ou les lasers excimères, a trouvé place dans le domaine industriel. Le principal avantage découlant de l'utilisation de ces sources réside dans le fait qu'avec des impulsions courtes (autour de $10 \mathrm{~ns}$ ) la matière irradiée peut être ablatée avec très peu d'effets thermiques en comparaison de ceux produits par des lasers continus ou à impulsions longues. Dans la majorité des cas, ce mécanisme ablatif se traduit par une précision accrue en ce qui concerne la profondeur de matière enlevée, ce qui fait de l'usinage laser un outil prometteur dans les domaines de la microélectronique, micro-optique et micro-mécanique. Les applications courantes de ce type de sources sont : le nettoyage des surfaces, la structuration des matériaux, le micro-perçage, la gravure des couches minces, le micro-usinage d'optiques diffractives, etc.... Il y a cependant plusieurs inconvénients liés à l'utilisation des lasers à impulsions courtes en ce qui concerne le micro-usinage des matériaux :

- La stabilité d'une impulsion à l'autre n'est pas toujours suffisante pour obtenir une bonne précision en ce qui concerne la profondeur de matière ablatée. Cette instabilité est particulièrement importante avec des émissions lasers obtenues par doublage ou triplage de fréquence de l'émission d'un laser à solide déclenché. 
- Avec certains lasers, en particulier les lasers excimères, le milieu actif se dégrade dans le temps et, de ce fait, les paramètres du procédé ne sont pas constants.

- L'irradiation laser peut causer une variation des propriétés physico-chimiques du matériau: L'absorption peut changer durant le procédé du fait de la modification de la rugosité ou de la composition chimique de la surface.

Bien que ces phénomènes aient été largement étudiés et compris, peu de procédés lasers industriels en tiennent compte. De ce fait, des ajustements délicats et fréquents sont nécessaires de façon à obtenir des résultats répétitifs. Pour l'automatisation d'un contrôle de procédé, un moyen de diagnostic temps réel est un auxiliaire utile à l'outil laser. Plusieurs techniques ont été développées. Un capteur piézo-électrique peut être fixé au dos de l'échantillon traité de façon à enregistrer l'onde de pression se propageant dans le matériau $\left.\left({ }^{1}\right),{ }^{2}\right)$. Un microphone et un système à déflexion de faisceau ont été utilisés pour détecter les variations de pression au-dessus de l'échantillon irradié $\left({ }^{3}\right),\left({ }^{4}\right)$.

Dans cette communication, nous décrivons l'utilisation de deux capteurs relativement simples qui permettent de suivre en temps réel le micro-usinage d'une céramique par laser.

\section{INTERACTION D'UN LASER A IMPULSION COURTE AVEC UN MATERIAU}

L'interaction entre une impulsion laser courte de haute fluence et différents matériaux a été largement décrite dans la littérature $\left.\left(^{5}\right),{ }^{6}\right)$. Les effets observés sont dus principalement à une évaporation intense du matériau. Les phénomènes de transport et les effets mécaniques sont alors prédominants.

La figure 1 résume les effets produits par l'irradiation d'un matériau par un laser à impulsions courtes. Le matériau absorbe l'énergie laser dans une très fine épaisseur d'environ une dizaine de nanomètres et qui peut varier suivant les matériaux. Du fait de la très haute irradiation (de l'ordre de $1 \mathrm{GW} / \mathrm{cm}^{2}$ ) la température d'évaporation est atteinte en quelques nanosecondes. La pression de vapeur augmente de façon significative durant la durée de l'impulsion. Une expansion supersonique des gaz peut alors survenir qui, en repoussant l'air ambiant est à l'origine de la formation d'une onde de choc dans celui-ci.

De la même façon, cette éjection à grande vitesse de matière vaporisée exerce au niveau de la pièce une force de recul qui se traduit par une onde de pression se propageant dans le matériau.

A haute fluence, $10 \mathrm{GW} / \mathrm{cm}^{2}$ ou plus suivant le matériau, les phénomènes d'ionisation dans la vapeur compliquent et rendent plus instable l'interaction. Le plasma ainsi cré́ peut devenir très absorbant pour le faisceau laser incident et peut faire écran vis-à-vis de la surface. En conséquence, une plus haute densité d'énergie ne se traduit pas forcément par un taux d'ablation plus élevé. Nos résultats expérimentaux montrent qu'il existe un domaine de fluence qui donnent un taux d'optimal optimum. 


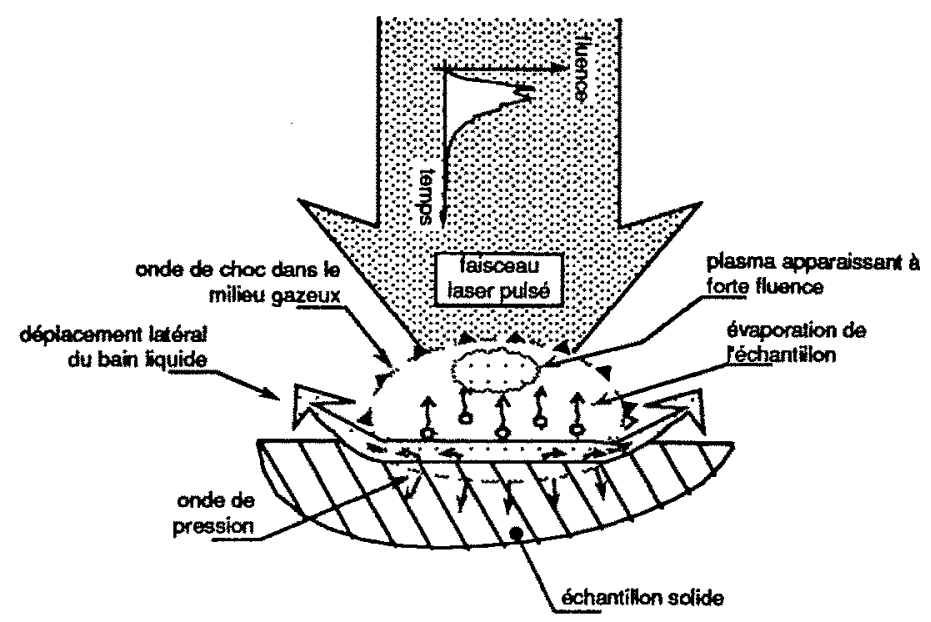

Figure 1 : Résumé des phénomènes se produisant lors de l'interaction entre un matériau et une impulsion laser d'une durée de l'ordre de la nanoseconde.

\section{MICRO-USINAGE LASER D'UNE CÉRAMIQUE}

L'usinage laser des céramiques a reçu récemment une importante attention due au fait que les céramiques, matériaux très durs ne peuvent être usinées facilement par des outils conventionnels. En particulier, l'usinage par des lasers à impulsions courtes présente l'avantage d'éviter les effets thermiques trop importants qui peuvent conduire à la formation de micro-fissures. Le dispositif expérimental est repris par la figure 2 . Le laser utilisé dans cette étude, est un laser excimère (modèle LUMONICS EX748). Ce laser produit des impulsions de $500 \mathrm{~mJ}$ de $15 \mathrm{~ns}$ de durée et à un taux de répétition maximum de $200 \mathrm{~Hz}$.

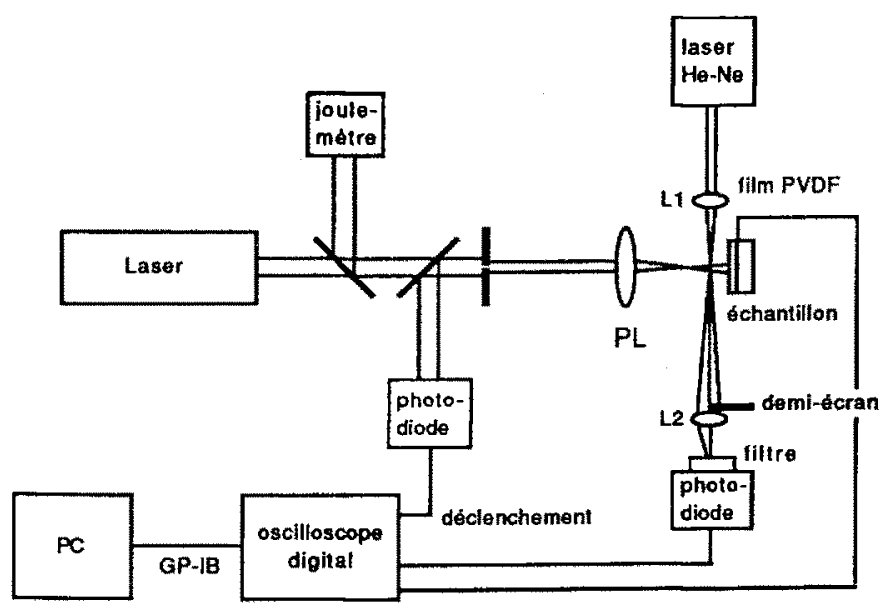

Figure 2 : Dispositif expérimental utilisé pour la caractérisation du procédé de micro-usinage de céramique par laser à impulsions courtes. 
Une partie homogène du faisceau a été sélectionnée par un masque et imagée sur la pièce à traiter par un objectif. Le diamètre de la tache irradiée est de l'ordre de $0.8 \mathrm{~mm}$. La fluence a pu être ajustée grâce à un atténuateur à lames de quartz et, déterminée par un joule mètre GENTEC ED 500.

Les fortes ondes de choc qui se propagent du point d'interaction dans le solide et dans l'air ambiant ont été détectées et mesurées par un capteur piézo-électrique et par la déflexion d'un faisceau sonde hélium-néon. Le capteur piézo-électrique consiste en un film de $9 \mu \mathrm{m}$ d'épaisseur de PVDF collé au dos d'un échantillon d'alumine pure à $99 \%$. Le faisceau sonde d'un laser hélium-néon de $5 \mathrm{~mW}$ passant parallèlement au-dessus de la surface de l'échantillon traité et, à une distance d'à peu près $15 \mathrm{~mm}$ de celle-ci, est dévié par le gradient d'indice que représente le passage de l'onde de choc crée lors de linteraction. Cette déflexion est enregistrée par un demi-écran et une photodiode. Une autre photodiode recevant une partie du faisceau assure un déclenchement stable de l'acquisition.

\section{MODÉLISATION DE LA PROPAGATION DE L'ONDE DE CHOC}

Le problème idéalisé d'une forte explosion dans une atmosphère homogène a déjà été traité par SEDOV ${ }^{7}$ ) qui a considéré un gaz parfait possédant une chaleur spécifique et une densité constante. Ce modèle d'explosion ponctuelle a également pour hypothèse le fait qu'une importante quantité d'énergie est libérée dans un faible volume et durant un intervalle de temps très court. Lorsqu'une impulsion laser de faible durée est focalisée sur un matériau solide, des particules sont éjectées de la surface à une vitesse supersonique: l'air ambiant est repoussé brusquement et l'énergie des particules est transférée à une onde de choc dont l'origine apparaît ponctuelle depuis un point d'observation éloigné de la zone d'interaction. Les hypothèses du modèle d'explosion ponctuelle décrit par SEDOV $\left({ }^{7}\right.$ ) sont alors vérifiées. Cette modélisation est utile car elle permet d'évaluer la quantité d'énergie transférée du faisceau laser dans l'onde de choc. Plusieurs solutions existent suivant limportance de l'onde de choc et la position d'observation. Dans le domaine où nous nous plaçons (environ $15 \mathrm{~mm}$ de la pièce) et vues les énergies laser envisagées (de 5 à $50 \mathrm{~mJ}$ ) la pression atmosphérique n'est plus négligeable ( ${ }^{8}$ ) vis-à-vis de la surpression de l'onde de choc. Dans ce domaine d'onde de choc faible, la solution au problème de propagation ne peut être obtenue que par une intégration numérique des equations différentielles appropriées. Les résultats de ces calculs sous formes de tables et de graphes peuvent être trouvés dans les références $\left({ }^{7}\right),\left({ }^{9}\right)$ et $\left({ }^{10}\right)$. Ces données nous ont permis de tracer la courbe de la figure 3 donnant la relation entre énergie de l'onde de choc et temps de transit de la surface de l'échantillon à la position d'observation.

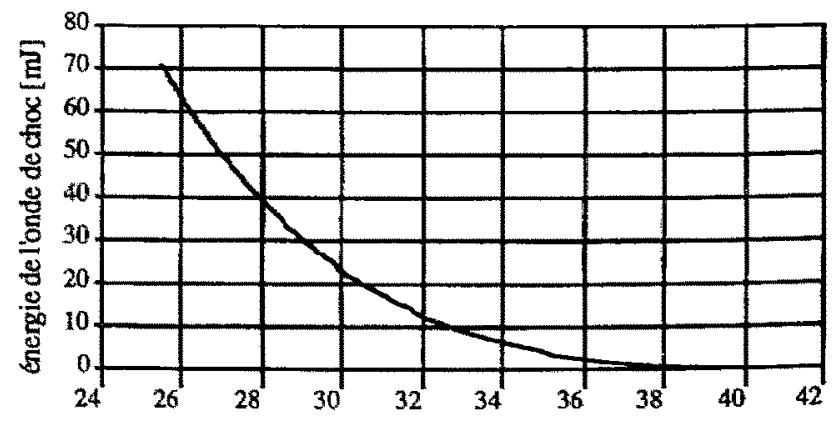

lemps de transit [us]

Figure 3 : Énergie de l'onde de choc en fonction de son temps de transit de l'échantillon a la position d'observation à $15 \mathrm{~mm}$. 


\section{RÉSULTATS EXPÉRIMENTAUX AVEC LE LASER KrF}

Les figures $4 \mathrm{a}$ et $4 \mathrm{~b}$ montrent respectivement la réponse du capteur piézoélectrique et l'énergie de l'onde de choc (calculée suivant la modélisation précédente) en fonction de la fluence laser. A partir de ces données on peut remarquer qu'il existe un seuil pour ces signaux qui correspond au seuil d'ablation (voir figure 5). Du point de vae du contrôle de procédé, les relations intéressantes sont celles qui lient les sigraux enregistrés à la quantité de matière ablatée (figure $6 \mathrm{a}$ et 6b).

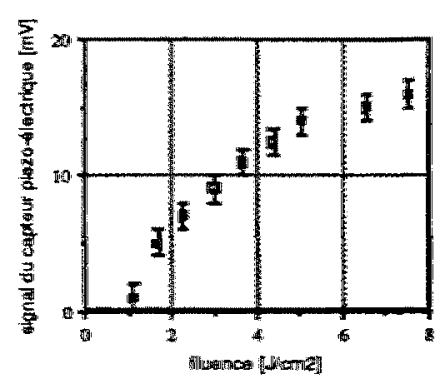

(a)

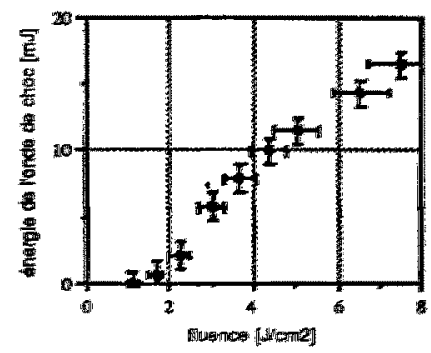

(b)

Fügure 4 : Contrôle da procédé d'usinage d'une céramique de type alumine par laser excimère.

(a) Réponse du capteir piézo-électrique;

(b) Énergie de l'onde de choc évaluée par la mesure du temps de transit.

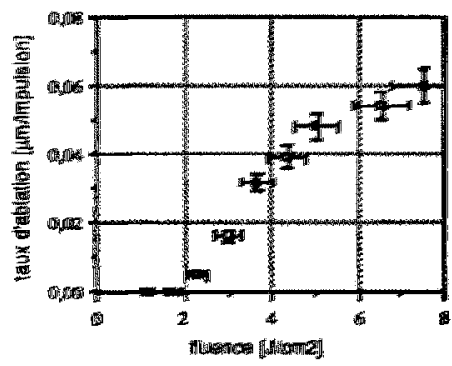

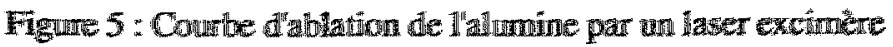

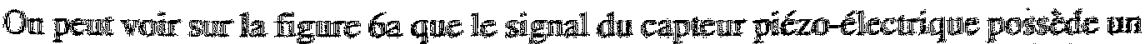
seuil en atessons duquell tahlation est quasii nulle. Au dessus de ce seuill, le signall obtenu

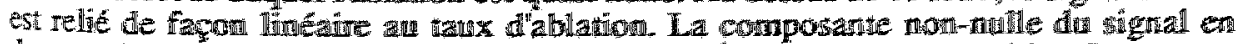

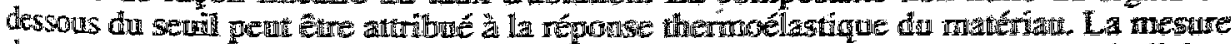

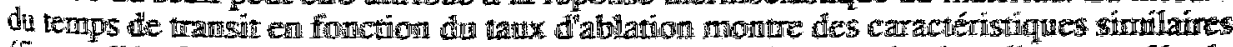
(figure 6b). Ce type de réppouse permet d'envisager l'antonatisation d"uin contrôle de

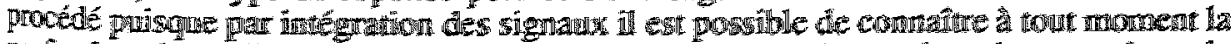

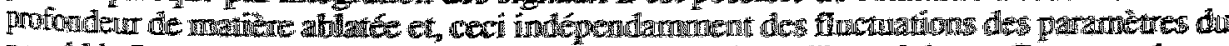
procédé. La paécission obtemte dêpend du nombre d"irmpullsions. Pour quelques

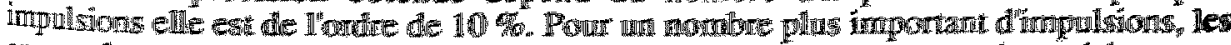

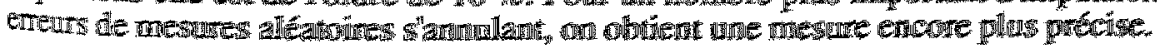




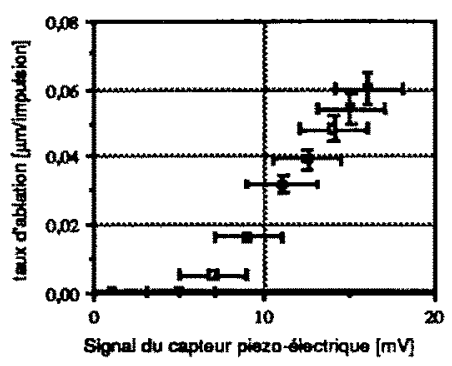

(a)

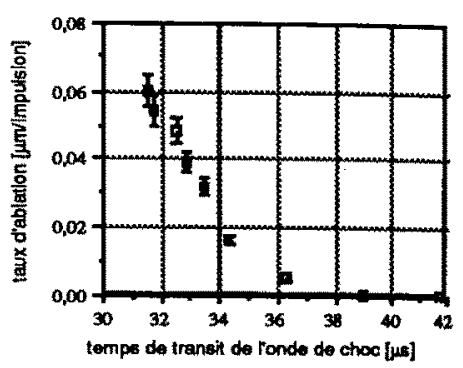

(b)

Figure 6: Relation entre le taux d'ablation d'alumine et

(a) le signal du capteur piézo-électrique;

(b) le temps de transit de l'onde de choc.

\section{RÉSULTATS EXPÉRIMENTAUX AVEC LE LASER ND:YAG DÉCLENCHÉ}

A titre de comparaison, des essais ont été menés sur le même type de matériau et, avec le même dispositif expérimental que celui décrit par la figure 2, avec un laser Nd:YAG déclenché (BMI 502-DNS 7730) dont la longueur d'onde d'émission se situe à $1,06 \mu \mathrm{m}$, dans le proche infrarouge. Les impulsions délivrées par ce laser ont une durée d'environ $15 \mathrm{~ns}$ et une énergie maximale de $750 \mathrm{~mJ}$.

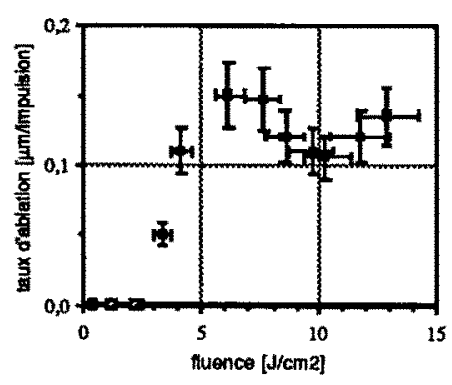

(a)

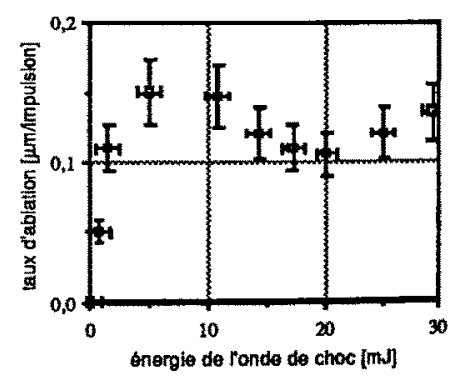

(b)

Figure 7 : Relation entre le taux d'ablation d'alumine et $7 \mathrm{a}:$ la fluence du laser Nd:YAG déclenché incidente. $7 \mathrm{~b}:$ l'énergie de l'onde de choc.

La figure 7a représente la variation du taux d'ablation en fonction de la fluence laser incidente sur la pièce d'alumine. On peut remarquer que le taux d'ablation de ce matériau présente un seuil en fonction de la fluence incidente proche de $2 \mathrm{~J} / \mathrm{cm}^{2}$, puis il augmente assez rapidement jusqu'à $6 \mathrm{~J} / \mathrm{cm}^{2}$ où il atteint la saturation. La technique de déflexion de faisceau qui nous permet de calculer l'énergie de l'onde de choc en fonction du temps de transit, est inadéquate au-delà de d'une fluence d'environ 5 à $6 \mathrm{~J} / \mathrm{cm}^{2}$ au contrôle de procédé. En effet, on peut voir sur la figure $7 \mathrm{~b}$ (à comparer avec la figure 8 qui correspond au cas du laser excimère) que la relation entre le taux d'ablation et l'énergie de l'onde de choc n'est plus univoque au-delà de cette fluence. Ces différents phénomènes que l'on ne rencontre pas dans le cas du traitement par laser excimère 
peuvent être attribués à la formation d'une onde de détonation lors de l'interaction. Le dispositif de projection utilisé est en effet, favorable à la formation d'une telle onde puisque le point de focalisation se trouve au-dessus de la pièce. De plus, le coefficient d'absorption variant avec le carré de la longueur d'onde, il est nettement plus important à $1,06 \mu \mathrm{m}$ qu' a $0,248 \mu \mathrm{m}$. Ainsi, la formation de cette onde de détonation, en faisant écran à l'irradiation laser, capte la plus grande partie de l'énergie délivrée par le laser. Ceci se traduit, d'une part, par une stagnation du taux d'ablation et, d'autre par le fait que l'énergie de l'onde de choc n'est plus conélée à la masse des débris, car augmentée d'une contribution issue du claquage de la vapeur.

\section{DISCUSSION}

Il est intéressant de vérifier la validité du modèle utilisé pour calculer l'énergie de l'onde de choc. Pour cela, on peut, connaissant les propriétés du matériau (alumine pure à $99 \%$ ) et, en s'appuyant sur les mesures de réflexivité réalisées par TÔNSHOFF (11) établir une ébauche de bilan énergétique de l'interaction alumine-laser excimère. A environ $4 \mathrm{~J} / \mathrm{cm}^{2}$, soit juste après le seuil d'ablation, le taux d'absorption mesuré par TÖNSHOFF est de 90 a $95 \%$, soit une énergie laser d'environ $15 \mathrm{~mJ}$. La profondeur d'absorption du rayonnent est d'environ $0.3 \mu \mathrm{m}$. Un calcul assez simple nous permet alors d'établir un bilan énergétique :

- énergie requise pour vaporiser $0.04 \mu \mathrm{m}$ d'alumine : $2 \mathrm{~mJ}$;

- énergie nécessaire à la formation d'une zone fondue de $0.5 \mu \mathrm{m}$ d'épaisseur : $2 \mathrm{~mJ}$;

- énergie calorifique diffusée dans le matériau : 2 à $3 \mathrm{~mJ}$;

Comme on mesure à cette fluence une énergie d'onde de choc d'à peu près $8 \mathrm{~mJ}$, on voit que le bilan est assez bien équilibré. La modélisation de l'onde de choc semble correcte. Si on refait un calcul analogue au alentour de $8 \mathrm{~J} / \mathrm{cm}^{2}$ on ne retrouve pas la même situation : il manque une dizaine de millijoules dans le bilan énergétique pour arriver aux $30 \mathrm{~mJ}$ apportées par le laser. On peut expliquer ce phénomène si on tient compte de l'émission lumineuse du plasma. En effet, à $4 \mathrm{~J} / \mathrm{cm}^{2}$, soit juste au-dessus du seuil, la majeure partie de l'évaporation se fait en fin de pulse et, par conséquent, les produits de l'ablation interagissent peu avec le faisceau. Par contre a $8 \mathrm{~J} / \mathrm{cm}^{2}$, lévaporation se produit plus tôt, les produits d'ablation interagissent avec le faisceau ce qui conduit à une perte d'énergie plus importante par émission lumineuse du plasma. Une étude quantitative ultérieure de l'émission plasma devrait confirmer ce fait.

Un autre fait intéressant que lon peut constater est la différence de mécanisme dinteraction laser-matière qui existe entre le laser excimère et le laser $\mathrm{Nd:YAG} \mathrm{déclenché}$ lors du traitement de l'alumine. Dans le premier cas, l'alumine absorbe le rayonnement UV dans une très fine épaisseur inférieure au micron, tandis que dans le second la profondeur d'absorption est d'environ une centaine de microns. Cela se traduit respectivement par une évaporation de la surface et par une ébullition explosive.

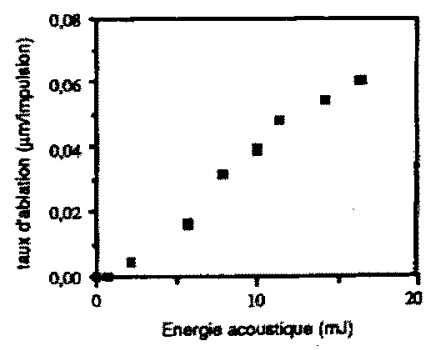

Figure 8 : Relation entre le taux d'ablation d'alumine par laser excimère et l'énergie de l'onde de choc 
La figure 8 montre la variation de l'énergie de l'onde de choc en fonction du taux d'ablation dans le cas du traitement de l'alumine par le laser excimère. On constate qu'il existe entre ces deux grandeurs une relation quasi-linéaire. C'est une constatation intéressante, car si on suppose comme dans la référence (12) que l'énergie de l'onde de choc est égale à l'énergie cinétique des débris, on trouve une vitesse moyenne d'éjection de l'ordre de $14 \mathrm{~km} . \mathrm{s}^{-1}$, en bon accord avec la référence $\left({ }^{13}\right)$. En ce qui concerne l'irradiation de l'alumine par le laser Nd:YAG déclenché, en utilisant la courbe de la figure $7 \mathrm{~b}$ en dessous de $5 \mathrm{~J} / \mathrm{cm}^{2}$, on peut voir que l'éjection d'une quantité assez importante de matière $(0.15 \mu \mathrm{m}$ d'épaisseur) se traduit par une énergie d'onde de choc faible $(5 \mathrm{~mJ})$. Ceci implique que les produits de l'ablation ont une vitesse assez faible (4 à $5 \mathrm{~km} . \mathrm{s}^{-1}$ ), ce qui est difficilement compatible avec une ablation uniquement en phase gazeuse. Des observations en microscopie électronique confirment ces hypothèses : on constate la présence de traînées d'alumine fondue, signe d'une ébullition explosive et d'éjection de matière en phase liquide.

\section{CONCLUSION}

Des lasers à impulsions courtes (Nd:YAG déclenché et excimère) ont été utilisés pour irradier une céramique de type alumine. L'interaction laser-matière est la source d'ondes de choc et de pression qui se propagent dans l'air et dans le matériau. L'observation de ces ondes par un capteur piézoélectrique et par une technique de déflexion de faisceau nous a permis d'évaluer en temps réel la quantité de matière ablatée lors du procédé. Dans le cas du traitement par laser Nd:YAG déclenché, il se produit à haute fluence un claquage de la vapeur qui conduit, d'une part à une saturation du taux d'ablation et, d'autre part, à une augmentation de l'énergie de l'onde de choc qui n'est plus alors corrélée avec la quantité de matière ablatée. On a également pu mettre en évidence les différences de mécanisme d'interaction suivant la longueur d'onde du laser utilisé. Au contraire du traitement par laser excimère où l'alumine est évacuée en phase gazeuse, le traitement par laser Nd:YAG déclenché conduit à une absorption volumique puis, à une surchauffe suivie d'une ébullition explosive.

\section{RÉFÉRENCES}

[1] Dyer P. E., Farrar S.R., and Key P.H., Appl. Surf. Science 54 (1992), 255-263.

[2] Ghost A.P., and Hurst J.E., J. Appl. Phys. 64,1 (1988), 287-290.

[3] Sell J.A., Heffelginger D., Ventzek P., and Gilgenbach R., J. Appl. Phys. 69,3 (1991), 1330-1335.

[4] Ventzek P., Gilgenbach R., Heffelginger D., and Sell J., J. Appl. Phys. 70,2 (1991), 587-593.

[5] Ready J.F., Effects of High-Power Laser Radiation, (Academic Pess, New York, 1971).

[6] Von Allmen M., Laser-Beam Interactions with Materials, (Springer-Verlag, Berlin, 1986).

[7] Sedov L. I., Similarity and Dimensional Methods in Mechanics, (Academic Press, New York, 1959).

[8] Zel'dovich Y.B. and Raizer Y.P., Physics of Shock Waves and High-Temperature Hydrodynamic Phenomena, (Academic Press, New York, 1966).

[9] Goldstine H. and Von Neumann J., Com.in Pure Appl. Math.8 (1955),327-353.

[10] Okhotsimskii I.L., Kondrasheva Z.P., Vlasova R.K., and Kasakova R.K., Calculation of a point explosion with counterpressure, Tr. Mat. Inst. Nauk. (1957).

[11] Tönshoff H.K. and Gedrat O.,"Absorption behaviour of ceramic materials irradiated with excimer lasers", Boston 8-9 November 1990 (SPIE proceedings vol. 1377).

[12] Rothenberg J.E. and Koren G. , Appl. Phys. Lett 44,7 (1984), 664-666.

[13] Dreyfus R. W. and Kelly R., Appl. Phys. Lett 49,21(1986), 1478-1480. 